\title{
EFECTO DE LA SUPLEMENTACIÓN CON MG SOBRE DIVERSOS PARÁMETROS EN VACAS CON RESTRICCIÓN ALIMENTARIA\#
}

\author{
THE EFFECT OF MAGNESIUM SUPPLEMENTS ON SEVERAL PARAMETERS CATTLE \\ UNDER NUTRITIONAL RESTRICTION
}

\author{
Cseh, S.B. ${ }^{\text {* }}$, Rodríguez García, M.J. ${ }^{2}$, Sciotti, A. ${ }^{2}$ y Campero, C.M. ${ }^{1}$
}

\begin{abstract}
'Estación Experimental Agropecuaria Balcarce. Instituto Nacional de Tecnología Agropecuaria. Buenos Aires. Argentina. *scseh@balcarce.inta.gov.ar

${ }^{2}$ Facultad de Ciencias Agrarias de Balcarce. Universidad Nacional de Mar del Plata. Balcarce. Buenos Aires. Argentina.
\end{abstract}

\section{Palabras clave adicionales}

Bovino. Hipomagnesemia. Restricción nutricional. Suplementación mineral.

\section{RESUMEN}

Se evaluó el efecto de la suplementación oral con Mg sobre la concentración intra y extracelular de macroelementos en vacas de cría con restricción nutricional durante el último tercio de gestación y 45 días posparto y determinar su influencia sobre parámetros bioquímicos y el estado clínico y productivo de los animales.

Se utilizaron tres grupos de 15 vacas Abeerden Angus, preñadas. El grupo 1 fue suplementado con óxido de magnesio con libre acceso a una pastura de agropiro y festuca; el grupo 2 no recibió suplementación mineral y tuvo acceso a las pasturas; en el grupo 3, los animales fueron sometidos a restricción alimentaria en el preparto, con acceso sólo a heno de agropiro. Se realizaron 6 muestreos de sangre, forraje y uno de agua de bebida. En suero sanguíneo, eritrocitos y forraje se midió Mg, Ca, Na y K por espectrofotometría de absorción atómica. En suero y pasto $\mathrm{P}$ por colorimetría. En agua $\mathrm{Ca}, \mathrm{Mg}$, Na por espectrofotometría de absorción atómica y sales totales por gravimetría. Los datos fueron analizados mediante ANOVA y comparados por el test de Duncan para cada grupo según muestreos. Las concentraciones de $\mathrm{Mg}$ en las pasturas y en heno

"El presente trabajo se financió con fondos provenientes del LBCV a través del Servicio de Diagnóstico Veterinario Especializado del INTA de Balcarce.

\section{AdDitiOnAL KEYWORDS}

Bovine. Hypomagnesemia. Mineral supplementation. Nutritional restriction.

fueron inferiores al $0,20 \%$ sobre materia seca. No existieron diferencias significativas en los valores del Mg sérico entre los 3 grupos de animales si bien el grupo 1 presentó valores más elevados. La concentración de Mg intracelular fue menor que la del extracelular en todos los grupos y durante el posparto se observó un aumento de los valores de $\mathrm{Mg}$ eritrocitario en todos los animales. Durante el último muestreo, los animales de los grupos 1 y 2 mantenían una condición corporal de 6 mientras que los del grupo 3 tenían una condición de 4 . Los animales de los grupos 1 y 2 ganaron 26 y $16 \mathrm{~kg}$ respectivamente, mientras que los del grupo 3 perdieron $44 \mathrm{~kg}$. Los porcentajes de destete fueron de $93,3 \%$ para los grupos 1 y 2 y $66,6 \%$ para el grupo 3. El peso al nacer de los terneros del grupo 3 fue de $4 \mathrm{~kg}$ menos que el de los grupos 1 y $2(p<0,05)$. La caída de Mg sérico fue más rápida que la del eritrocitario por lo que es mejor indicador para detectar la deficiencia de Mg. Si bien, no se observaron diferencias significativas entre los parámetros bioquímicos analizados, el grupo de animales bajo restricción alimentaria tuvo mayores pérdidas productivas que los animales sin restricción. Aunque no se observaron signos clínicos vinculantes a la hipomagnesemia en los animales en restricción este tipo de manejo alimenticio puede predisponer a pérdidas productivas que deberán ser evaluadas al momento de definir dicha estrategia. 


\section{CSEH, RODRÍGUEZ GARCÍA, SCIOTTIY CAMPERO}

\section{SUMMARY}

The objective of this work was to evaluate the effect of oral $\mathrm{Mg}$ supplements on intracellular and extracellular macro element concentrations in breeding cows with nutritional restrictions between the last third of the gestational period up to 45 days after birth. Three groups of 15 pregnant Aberdeen Angus cows each, were used in this study. Group 1 received a magnesium oxide supplement in addition to free access to agropyron and fescue pastures. Group 2 did not receive mineral supplements but did have access to the pastures. Group 3 had nutritional restrictions during the pre-partum period, with access exclusively to agropyron hay. Six samples of blood, forage and drinking water were collected. Blood serum, erythrocytes and forages were subjected to atomic absorption spectrophotometry in order to determine $\mathrm{Mg}, \mathrm{Ca}, \mathrm{Na}$ and $\mathrm{K}$ levels. Phosphorus levels were determined through colorimetry in serum and grass samples. Drinking water was used to measure $\mathrm{Ca}, \mathrm{Mg}$ and $\mathrm{Na}$ through atomic absorption spectrophotometry and total salt levels were obtained through gravimetric analysis. The data set was analyzed through ANOVA and groups were compared using Duncan's Test (MRT). Magnesium concentrations in pastures and hay were below $0.20 \%$ dry matter. No significant differences were observed on serum $\mathrm{Mg}$ levels among groups, although group 1 presented the highest levels throughout the study. Intracellular $\mathrm{Mg}$ levels were consistently lower than extracellular levels in all groups. During postpartum, erythrocytes magnesium levels increased for all groups. At the time of the last sample collection, animals from groups 1 and 2 exhibited a body condition value of 6 while group 3 presented a body condition value of 4 . The animals from groups 1 and 2 gained $26 \mathrm{~kg}$ and $16 \mathrm{~kg}$ respectively while those from group 3 lost $44 \mathrm{~kg}$. Weaning percentages were $93.3 \%$ for groups 1 and 2 and $66.6 \%$ for group 3 . Weights at birth for calves belonging to animals in group 3 were lower $(<4 \mathrm{~kg})$ than those belonging to groups 1 and $2(p<0.05)$. The magnesium decrease in plasma occurred earlier than the erythrocytes magnesium decrease. This suggests that plasma magnesium measurements are a more sensitive and earlier indicator to detect significant $\mathrm{Mg}$ level decrease than intracellular measurements. No significant differences were observed among the analyzed biochemical parameters. However, group 3 presented more productive losses than group 1 and 2. Even though clinical sign linked to hypomagnesaemia were not observed in group 3 , this type of nutritional management may encourage productive losses that must be evaluated at the time of implementing such a nutritional strategy.

\section{INTRODUCCIÓN}

El magnesio es indispensable para el normal funcionamiento del metabolismo animal (Suttle, 2010). En los bovinos, el Mg de los glóbulos rojos presenta menor concentración que el del suero $(0,68 \pm 0,10 \mathrm{mmol} /$ $1 ; 0,83 \pm 0,096 \mathrm{mmol} / 1$, respectivamente) no existiendo intercambio entre ambas fases en la circulación periférica (Cseh y Drake, 2000). La concentración de este mineral en el suero de los rumiantes está controlada por la absorción a partir de la dieta, la excreción renal y la difusión a través de los tejidos blandos (Urdaz et al., 2003).

La tetania hipomagnesémica es provocada por una rápida disminución de la concentración de $\mathrm{Mg}$ en la sangre. La misma puede ser provocada por un aporte inadecuado en la dieta (Martens y Schweigel, 2000) o por una deficiente absorción desde el tracto digestivo (Laporte y Sykes, 2004).

Por otro lado, la labilidad del Mg circulante toma al animal adulto más vulnerable, dado que la movilización del $\mathrm{Mg}$ desde el tejido óseo o muscular es lenta y escasa. Esta circunstancia hace que al disminuir la concentración sanguínea, el animal dependa básicamente de la ingesta diaria de este mineral para evitar el cuadro de hipomagnesemia (Contreras, 2002a).

La mortandad debida a esta enfermedad, en vacas de cría en el sudeste de la provincia de Buenos Aires, Argentina, ha sido estimado en 4-5\% (Cseh y Crenovich, 1996). Los factores de riesgo involucrados en la presentación de la misma están asociados al pastoreo de verdeos de invierno; prolongada ausencia a la exposición solar de los verdeos invernales acompañada de exceso de precipitaciones; pastoreo de rebrotes con alto contenido de agua, potasio, proteí- 


\section{SUPLEMENTACIÓN CON MG EN VACAS CON RESTRICCIÓN ALIMENTARIA}

nas y escasa fibra bruta, especialmente al comienzo de la primavera, condición corporal de los animales, estado fisiológico, edad, nivel de producción y tipo de manejo de los rodeos (Ledesma, 2008). Sin embargo, hembras gestantes pertenecientes a la Reserva Ganadera 6 (R6) del INTA Balcarce, Buenos Aires, sometidas a restricción alimenticia pero manteniendo adecuada condición corporal, rara vez han presentado cuadros de tetania por hipomagnesemia.

Los animales ubicados en este campo son sometidos a las siguientes pautas de manejo para aumentar su eficiencia de producción: entore restringido con un máximo de 60 días, en el momento de máxima oferta forrajera; destete entre los 5-6 meses; diagnóstico de preñez por tacto rectal eliminando las vacas vacías; refugo anual por desgaste dentario y por condiciones sanitarias; entore de vaquillonas con 15 meses en el mismo momento que se entoran las vacas adultas; restricción alimentaria otoño-invernal en las vacas preñadas; realimentación gradual previa al parto y ad libitum después de él; conservación de excedentes forrajeros otoñales en pie, como pasturas reservadas de otoño para el invierno y de excedentes primaverales, como heno, esta reserva se suministra a las vacas durante la restricción otoño- invernal, en la realimentación previa al parto y al inicio de la lactancia; aplicación rigurosa de medidas profilácticas y sanitarias de acuerdo con un calendario prefijado (Carrillo, 1997).

Los objetivos de este trabajo, fueron evaluar el efecto de la suplementación oral con $\mathrm{Mg}$ sobre la concentración intra y extracelular de $\mathrm{Mg}$, Ca, $\mathrm{P}$, Na y $\mathrm{K}$ en vacas de cría sometidas a restricción nutricional inducida en el último tercio de gestación y cuarenta y cinco días posparto y determinar la influencia de esta restricción nutricional transitoria sobre la ganancia de peso de las madres, el porcentaje de parición y destete, la presencia de abortos, el peso de los terneros al nacer y al destete, la ganancia de peso diaria y la mortandad de terneros.

\section{MATERIAL YMÉTODOS}

\section{ANIMALES Y MANEJO}

El ensayo se realizó en la (R6) del INTA Balcarce ( $37^{\circ} 45^{\prime} \mathrm{S} ; 58^{\circ} 17$ 'E) durante los meses de mayo a octubre del año 2007. El clima de la región es templado, subhúmedo con una precipitación de $850 \mathrm{~mm}$ y temperatura media anual de $13 / 16^{\circ} \mathrm{C}$.

Se utilizaron 45 vacas adultas de raza Aberdeen Angus, preñadas (5-6 meses de gestación), homogéneas desde el punto de vista de manejo y alimentación. Los animales tenían una condición corporal (CC) de 6 (escala de 1 a 9), (David y Morrow, 1986) en un rodeo con $90 \%$ de destete en los últimos 30 años.

Los animales se asignaron al azar en tres grupos de 15 vacas cada uno. El grupo $1(\mathrm{G} 1)$ en pastoreo directo suplementados oralmente con sales de magnesio, sin restricción forrajera. El grupo 2 (G2) en pastoreo directo sin suplementación mineral. El grupo 3 (G3) bajo restricción alimentaria, sin suplementación mineral. La restricción alimentaria consistió en el consumo de $9 \mathrm{~kg}$ de heno/vaca/día, durante el período preparto. Se registró mensualmente el peso vivo de todos los animales, se realizaron observaciones diarias del estado clínico, registros de abortos, mortalidad, tipo de parto (normal o distócico), porcentaje de parición y destete, el peso de los terneros al nacer y al destete, ganancia de peso diaria y mortandad de terneros.

La base forrajera de la R6 corresponde a pasturas cultivadas de agropiro (Thynopirum ponticum) y festuca (Festuca arundinacea) de más de veinte años de producción, implantadas sobre suelos ganaderos con adecuada concentración de P. El manejo de la restricción nutricional otoño-invernal de los vientres adultos, es una práctica implementada hace más de treinta años y forma parte del protocolo de manejo nutricional del rodeo. Durante el presente ensayo se realizaron seis muestreos de sangre en los animales y la misma cantidad en 


\section{CSEH, RODRÍGUEZ GARCÍA, SCIOTTIY CAMPERO}

el pasto $(29 / 05,26 / 06,01 / 08,03 / 09,02 / 10$ y 15/10). También se hizo un muestreo del agua que consumían los animales al inicio del ensayo.

\section{SUPLEMENTOMINERAL}

Se formuló una mezcla mineral compuesta por $50 \mathrm{~kg}$ de cloruro de sodio, $0,150 \mathrm{~kg}$ de sulfato de zinc comercial; $0,150 \mathrm{~kg}$ de sulfato de cobre; $12 \mathrm{~kg}$ de óxido de magnesio comercial y $12 \mathrm{~kg}$ de conchilla molida. El consumo diario de los animales se estimó en $15 \mathrm{~g}$ de $\mathrm{Mg}$ /animal/día al inicio del ensayo.

A partir del 18/09 se comenzó a suministrar la mitad de la dosis de $\mathrm{Mg}$ en la mezcla mineral debido a que los animales no la consumían por la mala palatabilidad que le confería el Mg. Durante el $2^{\circ}$ muestreo, una vez por día, se le agregó al suplemento mineral maíz en grano quebrado a razón de $50 \mathrm{~g}$ /animal mezclado en los mismos comederos para favorecer la palatabilidad del suplemento, suministrándose al final del ensayo un total de $150 \mathrm{~g} / \mathrm{animal} /$ día entre el suplemento mineral y el maíz.

\section{ANÁLISIS EFECTUADOS}

Forraje: las muestras se recolectaron manualmente (hand-plucking), (Langlands, 1974) en cada uno de los lotes utilizados por los animales. Las mismas fueron secadas en estufa a $60^{\circ} \mathrm{C}$ y mineralizadas con una mezcla de ácido nítrico y perclórico (2/2) (v/v). Las concentraciones de $\mathrm{Mg}, \mathrm{Ca}, \mathrm{Na}$ y $\mathrm{K}$ se determinaron mediante espectrofotometría de absorción atómica (EAA) (Perkin Elmer, 1982). El P se cuantificó por el método de Chem et al. (1959). Se calculó la relación K/ $(\mathrm{Ca}+\mathrm{Mg})$. No se pudieron obtener muestras de heno del muestreo $\mathrm{N}^{\circ} 6$ ya que las malas condiciones climáticas impidieron el ingreso al potrero.

Agua: se cuantificó Ca, $\mathrm{Mg}$ y Na por EEA y contenido de sales totales por gravimetría.

Sangre: las muestras fueron obtenidas por punción de la vena yugular recolectándose dos especímenes por animal: 1) Sangre con anticoagulante heparina, para determi- nar $\mathrm{Mg}, \mathrm{Ca}, \mathrm{Na}$ y $\mathrm{K}$ en los eritrocitos por EAA previa hemólisis de los mismos con una solución hipotónica. 2) Sangre sin anticoagulante para cuantificar: $\mathrm{Mg}, \mathrm{Ca}, \mathrm{Na}$ y K en el suero mediante EAA. Este análisis se realizó en un equipo Perkin Elmer 5100 PC utilizando cloruro de lantano como estabilizador para el $\mathrm{K}$ y $\mathrm{Ca}$, y cloruro de potasio para el Na (Perkin Elmer, 1982). Fósforo sérico con un espectrofotómetro Coleman Junior II según técnica de Cseh et al. (1994).

Estadístico: se utilizó un diseño experimental factorial con tres tipos de alimentación diferentes para los distintos tratamientos en los 6 muestreos, para cada mineral a determinar. Los datos fueron analizados mediante ANOVA y se utilizó el test de comparaciones múltiples Duncan con un nivel de confianza del $95 \%$ para establecer diferencias significativas entre tratamientos. Los datos fueron analizados según el programa MIXED del SAS (1995).

\section{RESULTADOS}

\section{FORRAJES}

Las concentraciones de $\mathrm{Mg}, \mathrm{Ca}$ y $\mathrm{P}$ en las pasturas y heno nunca alcanzaron a cubrir los requerimientos de los animales $(0,20 ; 0,40$ y $0,24 \%$ de MS, respectivamente) mientras que los niveles de $\mathrm{Na}$ y $\mathrm{K}$ fueron adecuados $(\mathrm{Na}>0,10 \% ; \mathrm{K}<2,5 \%$ de $\mathrm{MS}$ ) (NRC, 2000) (tabla I).

En los análisis del forraje consumido por los animales de los G1 y G2 en el muestreo 5 se estableció que la relación $\mathrm{K} /(\mathrm{Ca}+\mathrm{Mg})$ fue >2,2 nivel considerado como tetanizante (Kemp y t'Hart, 1957). Para el resto de las determinaciones, la relación fue $<2,2$.

En el agua de bebida el contenido de sales totales fue de $591 \mathrm{mg} / \mathrm{L} ; \mathrm{Mg} 10 \mathrm{mg} / \mathrm{L}$; Ca 5 mg/L y Na 197 mg/L. Este agua resultó potable para el consumo animal, pero con bajo aporte de minerales.

\section{SuEROSANGUíNEO}

Los valores séricos promedio de $\mathrm{Mg}, \mathrm{Ca}$, $\mathrm{P}, \mathrm{K}$ y Na según tratamientos y muestreos se 


\section{SUPLEMENTACIÓN CON MG EN VACAS CON RESTRICCIÓN ALIMENTARIA}

presentan en la tabla II.

Para los animales del G1, los valores de Mg siempre estuvieron dentro del rango de referencia: 0,80-1,33 mmol/l(Whitaker, 2000;

Tabla I. Concentración (\% MS) de Mg, Ca, $P, K$ y Na en el alimento según tratamientos: G1 animales suplementados con magnesio y libre acceso a pastura; $G 2$ animales sin suplementación mineral y con acceso a pasturas; 63 animales sometidos a restricción alimentaria en el preparto, con acceso sólo a heno de agropiro. (Concentration (\%DM) of $\mathrm{Mg}, \mathrm{Ca}, \mathrm{P}, \mathrm{K}$, and $\mathrm{Na}$ in the food according to treatments: $G 1$ received a magnesium supplement in addition to free access to pasture; G2 did not receive mineral supplements but with access to the pastures; G3 had nutritional restrictions during the pre-partum period, with access exclusively to agropyron hay)

\begin{tabular}{|c|c|c|c|c|c|c|}
\hline \multirow[b]{2}{*}{ M1 } & \multirow[t]{2}{*}{$\mathrm{Mg}$} & \multirow[t]{2}{*}{$\mathrm{Ca}$} & \multirow[t]{2}{*}{$P$} & \multirow[t]{2}{*}{$\mathrm{K}$} & \multicolumn{2}{|c|}{$\mathrm{Na} \mathrm{K} /(\mathrm{Ca}+\mathrm{Mg})$} \\
\hline & & & & & & \\
\hline G1 & 0,16 & 0,096 & 0,13 & 1,69 & 0,62 & 0,70 \\
\hline G2 & 0,15 & 0,12 & 0,145 & 1,12 & 0,60 & 1,57 \\
\hline G3 & 0,10 & 0,086 & 0,095 & 0,81 & 0,42 & 1,66 \\
\hline \multicolumn{7}{|l|}{ M2 } \\
\hline G1 & 0,14 & 0,10 & 0,095 & 0,71 & 0,35 & 1,08 \\
\hline G2 & 0,17 & 0,11 & 0,095 & 0,94 & 0,52 & 1,83 \\
\hline G3 & 0,08 & 0,07 & 0,06 & 0,51 & 0,14 & 1,26 \\
\hline \multicolumn{7}{|l|}{ M3 } \\
\hline G1 & 0,12 & 0,15 & 0,182 & 1,12 & 0,21 & 1,66 \\
\hline G2 & 0,077 & 0,15 & 0,105 & 0,76 & 0,13 & 1,36 \\
\hline G3 & 0,047 & 0,068 & 0,07 & 0,34 & 0,16 & 0,23 \\
\hline \multicolumn{7}{|c|}{ M4 } \\
\hline G1 & 0,087 & 0,061 & 0,095 & 0,76 & 0,27 & 1,95 \\
\hline G2 & 0,10 & 0,255 & 0,20 & 1,42 & 0,14 & 1,70 \\
\hline G3 & 0,045 & 0,071 & 0,13 & 0,5 & 0,14 & 1,79 \\
\hline \multicolumn{7}{|c|}{ M5 } \\
\hline G1 & 0,082 & 0,13 & 0,22 & 1,20 & 0,20 & 2,32 \\
\hline G2 & 0,074 & 0,06 & 0,11 & 1,00 & 0,41 & 2,79 \\
\hline G3 & 0,07 & 0,19 & 0,15 & 0,37 & 0,10 & 0,63 \\
\hline \multicolumn{7}{|l|}{ M6 } \\
\hline G1 & 0,11 & 0,2 & 0,145 & 1,15 & 0,18 & 1,51 \\
\hline G2 & 0,082 & 0,075 & 0,16 & 0,82 & 0,50 & 1,99 \\
\hline G3 & & & $\operatorname{Sin} d$ & datos & & \\
\hline
\end{tabular}

$\mathrm{K} /(\mathrm{Ca}+\mathrm{Mg})$ : Potencial tetanizante (miliequivalentes). M: muestreo; G: grupo.
Merck, 2003). Si bien se notó una tendencia a que estos animales presentaran una magnesemia mayor durante el transcurso de los muestreos, la diferencia no fue estadísticamente significativa $(p>0,05)$ con los otros grupos. Esta tendencia se hizo más notoria durante el posparto.

Para los animales del G2, los valores de $\mathrm{Mg}$ fueron normales hasta el muestreo 4 inclusive, observándose una tendencia al descenso progresivo en el tiempo hasta llegar a valores de hipomagnesemia, aunque sin diferencias significativas. Para los animales de G3 (tabla II), con excepción del muestreo 2, la magnesemia siempre estuvo por debajo del valor de referencia.

En cuanto a los valores de Ca en los tres grupos, siempre estuvieron por debajo del valor normal (2,37-3,12 mmol/L) (Laboratorio de Bioquímica Clínica Veterinaria (LBCV), INTA Balcarce).

Las concentraciones de $\mathrm{P}$ para todos los grupos se mantuvieron normales a lo largo del ensayo (1,13 - 2,42 mmol/L) (LBCV, INTA Balcarce), si bien en el G3 la fosforemia presentó una tendencia a disminuir en el muestreo 4 en coincidencia con el período de parición.

El K y el Na en los tres grupos, se mantuvieron dentro del rango normal (5-6 mmol/ L y 132,17 - 152,17 mmol/L, respectivamente) (LBCV, INTA Balcarce). En lo referente al $\mathrm{Na}$, se detectó una disminución en los tres grupos en el muestreo 5.

\section{GLóbulos Rojos}

En el caso de los glóbulos rojos (tabla III) para el $\mathrm{Mg}$ se encontraron diferencias significativas en los tres grupos a partir del muestreo $4(\mathrm{p}<0,05)$. El Mg eritrocitario fue elevándose a lo largo de los muestreos, no ocurriendo lo mismo con el $\mathrm{Mg}$ sérico donde no se observaron variaciones entre grupos.

Si se analizan los grupos individualmente, los valores de $\mathrm{Mg}$ en los glóbulos rojos siempre fueron menores en los animales del G3. Los animales de los G1 y G2 excepto en los muestreo 5 y muestreo 6 , donde se de- 


\section{CSEH, RODRÍGUEZ GARCÍA, SCIOTTIY CAMPERO}

tectaron los mayores valores, no presentaron diferencias significativas entre ellos. Para el G3 los mayores valores también se encontraron en los muestreo 5 y 6.

Salvo en el Na, Mg, Ca y K, presentaron alguna diferencia significativa, tanto en el tiempo como entre tratamientos.

\section{PARÁmETROS PRODUCTIVOS}

Las vacas de los G1 y G2 ganaron peso durante los dos primeros meses posparto mientras que las vacas del G3 perdieron peso y condición corporal (escala de 6 a 3 ) (tabla IV). En este grupo, cuatro vacas abortaron y una tuvo un parto con asistencia con posterior muerte del ternero.

Los porcentajes de parición de los G1 y G2 fueron $100 \%$ mientras que en el G3 fue del $91 \%$. El porcentaje de destete fue para el G1 y el G2 del $97,8 \%$ y para el G3 del 88,9\%.

Tabla II. Valores séricos medios para $\mathrm{Mg}, \mathrm{Ca}, \mathrm{P}, \mathrm{K}$ y $\mathrm{Na}(\mathrm{mmol} / \mathrm{L})$ según muestreos y tratamientos: $G 1$ animales suplementados con magnesio y con libre acceso a pastura; $G 2$ animales sin suplementación mineral y con acceso a pasturas; G3 animales sometidos a restricción alimentaria en el preparto, con acceso sólo a heno de agropiro. (Average values for $\mathrm{Mg}, \mathrm{Ca}, \mathrm{P}, \mathrm{K}$ and $\mathrm{Na}(\mathrm{mmol} / \mathrm{L})$ according samplings and treatments: $\mathrm{G} 1$ received a magnesium supplement in addition to free access to pasture; G2 did not receive mineral supplements but did have access to the pastures; G3 had nutritional restrictions during the pre-partum period, with access exclusively to agropyron).

\begin{tabular}{|c|c|c|c|c|c|}
\hline & $\mathrm{Mg}$ & $\mathrm{Ca}$ & $P$ & $\mathrm{~K}$ & $\mathrm{Na}$ \\
\hline \multicolumn{6}{|l|}{ M1 } \\
\hline G1 & $0,78 \pm 0,08^{b}$ & $1,90 \pm 0,13^{a}$ & $1,89 \pm 0,23^{a}$ & $3,55 \pm 0,42^{a}$ & $133,76 \pm 18,53^{a}$ \\
\hline G2 & $0,84 \pm 0,06^{a}$ & $1,99 \pm 0,13^{a}$ & $1,86 \pm 0,35^{a}$ & $3,86 \pm 0,42^{a}$ & $135,50 \pm 19,07^{a}$ \\
\hline G3 & $0,70 \pm 0,06^{c}$ & $1,84 \pm 0,11^{b}$ & $1,71 \pm 0,24^{a}$ & $3,60 \pm 0,56^{a}$ & $113,62 \pm 16,32^{a}$ \\
\hline \multicolumn{6}{|l|}{ M2 } \\
\hline $\mathrm{G} 1$ & $0,83 \pm 0,05^{a}$ & $1,81 \pm 0,11^{b}$ & $1,45 \pm 0,39^{a}$ & $3,50 \pm 0,44^{b}$ & $148,92 \pm 22,73^{a}$ \\
\hline G2 & $0,83 \pm 0,06^{a}$ & $2,04 \pm 0,10^{a}$ & $1,44 \pm 0,22^{a}$ & $3,36 \pm 0,35^{b}$ & $125,01 \pm 27,45^{b}$ \\
\hline G3 & $0,75 \pm 0,06^{a}$ & $1,99 \pm 0,12^{a}$ & $1,36 \pm 0,22^{a}$ & $4,18 \pm 0,49^{a}$ & $127,13 \pm 13,70^{a}$ \\
\hline \multicolumn{6}{|l|}{ M3 } \\
\hline G1 & $0,80 \pm 0,06^{a}$ & $2,05 \pm 0,09^{a}$ & $1,48 \pm 0,24^{b}$ & $3,92 \pm 1,39^{\mathrm{a}}$ & $127,67 \pm 9,77^{a}$ \\
\hline G2 & $0,79 \pm 0,08^{a}$ & $1,96 \pm 0,13^{b}$ & $1,55 \pm 0,28^{a}$ & $3,24 \pm 0,98^{a}$ & $134,63 \pm 16,66^{a}$ \\
\hline G3 & $0,64 \pm 0,09^{b}$ & $1,61 \pm 0,10^{c}$ & $1,74 \pm 0,22^{a}$ & $3,55 \pm 0,88^{a}$ & $108,70 \pm 22,95^{b}$ \\
\hline \multicolumn{6}{|l|}{ M4 } \\
\hline G1 & $0,80 \pm 0,06^{a}$ & $1,70 \pm 0,21^{a}$ & $1,36 \pm 0,29^{a}$ & $5,98 \pm 1,06^{a}$ & $146,52 \pm 18,82^{a}$ \\
\hline G2 & $0,77 \pm 0,08^{a}$ & $1,65 \pm 0,17^{b}$ & $1,42 \pm 0,17^{a}$ & $4,42 \pm 0,48^{b}$ & $130,63 \pm 13,13^{b}$ \\
\hline G3 & $0,72 \pm 0,10^{b}$ & $1,82 \pm 0,21^{a}$ & $1,49 \pm 0,57^{a}$ & $4,87 \pm 0,82^{b}$ & $125,01 \pm 25,72^{b}$ \\
\hline \multicolumn{6}{|l|}{ M5 } \\
\hline G1 & $0,78 \pm 0,05^{a}$ & $1,77 \pm 0,19^{a}$ & $1,49 \pm 0,24^{b}$ & $4,76 \pm 0,44^{b}$ & $82,02 \pm 8,23^{b}$ \\
\hline G2 & $0,72 \pm 0,08^{a}$ & $1,63 \pm 0,34^{a}$ & $1,81 \pm 0,32^{\mathrm{a}}$ & $5,55 \pm 1,40^{a}$ & $86,09 \pm 10,25^{a}$ \\
\hline G3 & $0,70 \pm 0,07^{\mathrm{b}}$ & $1,77 \pm 0,17^{a}$ & $1,27 \pm 0,31^{b}$ & $3,87 \pm 0,75^{c}$ & $93,48 \pm 16,84^{a}$ \\
\hline \multicolumn{6}{|l|}{ M6 } \\
\hline G1 & $0,84 \pm 0,15^{a}$ & $1,66 \pm 0,20^{a}$ & $1,61 \pm 0,16^{a}$ & $5,43 \pm 0,36^{a}$ & $118,54 \pm 13,82^{a}$ \\
\hline G2 & $0,73 \pm 0,29^{a}$ & $1,79 \pm 0,13^{a}$ & $1,70 \pm 0,22^{a}$ & $5,45 \pm 0,29^{a}$ & $121,07 \pm 12,24^{a}$ \\
\hline G3 & $0,74 \pm 0,08^{a}$ & $1,77 \pm 0,17^{a}$ & $1,29 \pm 0,21^{b}$ & $4,93 \pm 0,45^{b}$ & $106,50 \pm 13,19^{b}$ \\
\hline
\end{tabular}

n: 15 animales; \pm : desvío estándar; M: muestreo; G: grupo. abcDentro de cada muestreo, letras distintas en columnas, indican diferencias significativas (Test de Duncan, $p<0,05$ ).

Archivos de zootecnia vol. 61, núm. 236, p. 530. 


\section{SUPLEMENTACIÓN CON MG EN VACAS CON RESTRICCIÓN ALIMENTARIA}

Los terneros nacidos de las vacas del G3 pesaron al nacer $23 \pm 3,32 \mathrm{~kg}$, los de los grupos $\mathrm{G} 1$ y G2 pesaron $27 \pm 4,19$ y $27 \pm 2,0 \mathrm{~kg}$, respectivamente. Esto corresponde, en el caso del G3 a $4 \mathrm{~kg}$ menos en promedio con respecto al peso de los terneros de los otros grupos $(\mathrm{p}<0,05)$.

En los grupos G1 y G2 se encontró un ternero muerto al nacer en cada uno.

Los pesos al destete de los terneros de los G1, G2 y G3 fueron de 165,5 $\pm 17,52 \mathrm{~kg}$; $144,7 \pm 16,67$ y $149,2 \pm 18,64 \mathrm{~kg}$, respectivamente, con diferencias significativas de G1 con respecto a $\mathrm{G} 2$ y G3 $(\mathrm{p}<0,05)$. La ganancia diaria de peso vivo $(\mathrm{g} / \mathrm{d})$ fue de $0,68 \pm 0,066$; $0,61 \pm 0,086$ y $0,63 \pm 0,062$ para $\mathrm{G} 1, \mathrm{G} 2$ y G3.

\section{DISCUSIÓN}

En las condiciones del presente trabajo se observó que si bien la concentración de

Tabla III. Valores promedio de $\mathrm{Mg}, \mathrm{Ca}, \mathrm{K}$ y $\mathrm{Na}$ (mmol/L) en glóbulos rojos según muestreos y tratamientos: G1 animales suplementados con magnesio y con libre acceso a una pastura; G2 animales sin suplementación mineral y con acceso a pasturas; G3 animales sometidos a restricción alimentaria en el preparto, con acceso sólo a heno de agropiro. (Average values for $\mathrm{Mg}, \mathrm{Ca}, \mathrm{K}$ and $\mathrm{Na}(\mathrm{mmol} / \mathrm{L})$ in red blood cells according to samplings and treatments: $\mathrm{G} 1$ received a magnesium supplement in addition to free access to pasture; G2 did not receive mineral supplements but did have access to the pastures; G3 had nutritional restrictions during the pre-partum period, with access exclusively to agropyron).

\begin{tabular}{ccccc}
\hline & $M g$ & $\mathrm{Ca}$ & $\mathrm{K}$ & $\mathrm{Na}$ \\
\hline M1 & & & & \\
G1 & $0,36 \pm 0,047^{\mathrm{a}}$ & $0,056 \pm 0,018^{\mathrm{b}}$ & $1,51 \pm 0,33^{\mathrm{b}}$ & $7,56 \pm 0,52^{\mathrm{a}}$ \\
G2 & $0,37 \pm 0,050^{\mathrm{a}}$ & $0,070 \pm 0,020^{\mathrm{a}}$ & $1,50 \pm 0,29^{\mathrm{b}}$ & $7,55 \pm 0,51^{\mathrm{a}}$ \\
G3 & $0,33 \pm 0,050^{\mathrm{a}}$ & $0,067 \pm 0,017^{\mathrm{b}}$ & $1,81 \pm 0,30^{\mathrm{a}}$ & $7,33 \pm 0,71^{\mathrm{a}}$ \\
M2 & & & \\
G1 & $0,37 \pm 0,049^{\mathrm{a}}$ & $0,028 \pm 0,011^{\mathrm{a}}$ & $1,61 \pm 0,43^{\mathrm{a}}$ & $7,30 \pm 0,51^{\mathrm{a}}$ \\
G2 & $0,38 \pm 0,060^{\mathrm{a}}$ & $0,036 \pm 0,040^{\mathrm{a}}$ & $1,77 \pm 0,38^{\mathrm{a}}$ & $7,85 \pm 0,90^{\mathrm{a}}$ \\
G3 & $0,33 \pm 0,063^{\mathrm{b}}$ & $0,035 \pm 0,036^{\mathrm{a}}$ & $1,87 \pm 0,53^{\mathrm{a}}$ & $7,42 \pm 1,09^{\mathrm{a}}$ \\
M3 & & & \\
G1 & $0,39 \pm 0,043^{\mathrm{a}}$ & $0,046 \pm 0,016^{\mathrm{a}}$ & $2,13 \pm 0,41^{\mathrm{a}}$ & $7,22 \pm 1,42^{\mathrm{a}}$ \\
G2 & $0,38 \pm 0,040^{\mathrm{a}}$ & $0,048 \pm 0,020^{\mathrm{a}}$ & $1,94 \pm 0,35^{\mathrm{a}}$ & $7,16 \pm 0,85^{\mathrm{a}}$ \\
G3 & $0,34 \pm 0,035^{\mathrm{a}}$ & $0,048 \pm 0,027^{\mathrm{a}}$ & $2,08 \pm 0,70^{\mathrm{a}}$ & $6,77 \pm 0,93^{\mathrm{a}}$ \\
M4 & & & \\
G1 & $0,37 \pm 0,037^{\mathrm{a}}$ & $0,036 \pm 0,023^{\mathrm{a}}$ & $1,57 \pm 0,32^{\mathrm{a}}$ & $7,18 \pm 0,70^{\mathrm{a}}$ \\
G2 & $0,39 \pm 0,050^{\mathrm{a}}$ & $0,040 \pm 0,010^{\mathrm{a}}$ & $1,64 \pm 0,27^{\mathrm{a}}$ & $7,32 \pm 0,62^{\mathrm{a}}$ \\
G3 & $0,34 \pm 0,033^{\mathrm{b}}$ & $0,057 \pm 0,025^{\mathrm{a}}$ & $1,80 \pm 0,65^{\mathrm{a}}$ & $7,25 \pm 0,84^{\mathrm{a}}$ \\
M5 & & & \\
G1 & $0,45 \pm 0,058^{\mathrm{a}}$ & $0,045 \pm 0,013^{\mathrm{a}}$ & $1,55 \pm 0,39^{\mathrm{a}}$ & $7,10 \pm 0,92^{\mathrm{a}}$ \\
G2 & $0,44 \pm 0,040^{\mathrm{a}}$ & $0,048 \pm 0,010^{\mathrm{a}}$ & $1,58 \pm 0,27^{\mathrm{a}}$ & $7,26 \pm 0,85^{\mathrm{a}}$ \\
G3 & $0,42 \pm 0,040^{\mathrm{a}}$ & $0,053 \pm 0,032^{\mathrm{a}}$ & $1,73 \pm 0,65^{\mathrm{a}}$ & $7,26 \pm 0,95^{\mathrm{a}}$ \\
M6 & $0,52 \pm 0,105^{\mathrm{a}}$ & $0,056 \pm 0,012^{\mathrm{a}}$ & $1,82 \pm 0,25^{\mathrm{a}}$ & $8,07 \pm 1,06^{\mathrm{a}}$ \\
G1 & $0,52 \pm 0,090^{\mathrm{a}}$ & $0,047 \pm 0,008^{\mathrm{b}}$ & $1,72 \pm 0,21^{\mathrm{a}}$ & $7,64 \pm 1,12^{\mathrm{a}}$ \\
G2 & $0,46 \pm 0,072^{\mathrm{a}}$ & $0,066 \pm 0,028^{\mathrm{a}}$ & $1,93 \pm 0,43^{\mathrm{a}}$ & $8,04 \pm 0,83^{\mathrm{a}}$ \\
G3 & & & &
\end{tabular}

n: 15 animales; \pm : desvío estándar; M: muestreo; G: grupo. abDentro de cada muestreo, letras distintas en columnas, indican diferencias significativas (Test de Duncan, $p<0,05$ ). 


\section{CSEH, RODRÍGUEZ GARCÍA, SCIOTTIY CAMPERO}

Tabla IV. Promedios de peso vivo ( $\mathrm{kg}$ ) y condición corporal $(C C)$ de las madres según muestreos y grupos: G1 animales suplementados con magnesio y con libre acceso a pastura; G2 animales sin suplementación mineral y con acceso a pasturas; G3 animales sometidos a restricción alimentaria en el preparto, con acceso sólo a heno de agropiro. (Averages for mothers live weight $(\mathrm{kg})$ and corporal condition $(\mathrm{CC})$ according to samplings and groups: $\mathrm{G} 1$ received a magnesium supplement in addition to free access to pasture; G2 did not receive mineral supplements but did have access to the pastures; G3 had nutritional restrictions during the pre-partum period, with access exclusively to agropyron).

\begin{tabular}{ccccccc}
\hline & Gl & CC & G2 & CC & G3 & CC \\
\hline M1 & $348,20 \pm 33,70^{\mathrm{a}}$ & 6 & $335,73 \pm 22,02^{\mathrm{a}}$ & 6 & $383,73 \pm 40,72^{\mathrm{a}}$ & 6 \\
M2 & $363,07 \pm 37,74^{\mathrm{a}}$ & 6 & $356,07 \pm 21,96^{\mathrm{a}}$ & 6 & $381,93 \pm 42,72^{\mathrm{a}}$ & 6 \\
M3 & $376,13 \pm 42,08^{\mathrm{a}}$ & 6 & $357,20 \pm 19,90^{\mathrm{a}}$ & 6 & $373,27 \pm 42,29^{\mathrm{a}}$ & 6 \\
M4 & $352,73 \pm 35,55^{\mathrm{a}}$ & 6 & $338,20 \pm 33,85^{\mathrm{a}}$ & 6 & $338,80 \pm 35,32^{\mathrm{b}}$ & 6 \\
M5 & $353,67 \pm 39,95^{\mathrm{a}}$ & 6 & $327,40 \pm 20,10^{\mathrm{a}}$ & 5 & $350,40 \pm 32,03^{\mathrm{a}}$ & 5 \\
M6 & $374,20 \pm 40,47^{\mathrm{a}}$ & 6 & $351,33 \pm 23,31^{\mathrm{a}}$ & 6 & $339,67 \pm 34,33^{\mathrm{b}}$ & 4 \\
\hline
\end{tabular}

ab Letras diferentes en columnas indican diferencias significativas $(p<0,05)$ entre muestreos $(M)$ para cada grupo $(G)$.

$\mathrm{Mg}$ en el alimento no llegó a cubrir los requerimientos mínimos sugeridos (NRC, 2000) para este mineral en los animales, estos nunca manifestaron signos clínicos de tetania por hipomagnesemia. Los valores de $\mathrm{Mg}$ en el alimento pueden ser atribuidos a las condiciones climáticas presentes, sobre todo durante los últimos 3 muestreos, cuando se registraron altas precipitaciones provocando probablemente un efecto de lavado del suelo con el consecuente lixiviado del Mg como sugiere Bould (1964).

Las concentraciones encontradas de $\mathrm{Mg}, \mathrm{Ca}$ y $\mathrm{Na}$ en el agua de bebida permiten inferir que el aporte de minerales a partir de esta fuente fue irrelevante.

El hecho de que la magnesemia del G1 fuera la única que presentara una tendencia a tener los valores más elevados puede ser explicado teniendo en cuenta lo expresado por algunos autores quienes consideran que el valor crítico de $\mathrm{Mg}$ en sangre para evidenciar la efectividad de la suplementación y obtener respuesta favorable al tratamiento debería ser $\leq 0,60 \mathrm{mmol} / \mathrm{L}$ (Allworth et al., 1985; Capen y Rosol, 1989). En este trabajo, la magnesemia nunca estuvo por debajo de esos valores. Por otra parte, la efectividad del animal para absorber $\mathrm{Mg}$ aumenta a medida que se incrementan sus requerimientos (Capen y Rosol, 1989). La menor magnesemia encontrada en el muestreo 1 para el G1 podría deberse a que los animales se negaban a ingerir la mezcla mineral por su mala palatabilidad. A partir del muestreo 2, los animales comenzaron a comer una mayor cantidad por la adición de maíz. La suplementación con $\mathrm{MgO}_{\text {ó }} \mathrm{MgSO}_{4}$ es uno de los recursos empleados para corregir los problemas de hipomagnesemia (Odette, 2005; Scandolo, 2005). La dosis de Mg comúnmente utilizada para la suplementación en vacas para carne con riesgo de tetania es de $20 \mathrm{~g} /$ día (Scandolo, 2005). La cantidad suministrada al final del ensayo entre el suplemento y el maíz, fue suficiente para cubrir los requerimientos, lo cual explicaría los niveles normales de $\mathrm{Mg}$ en el suero a lo largo del ensayo. El mayor descenso de la magnesemia en el G2 en los muestreo 5 y muestreo 6 podría ser debido al efecto de la lactancia, ya que a partir del muestreo 4, el $80 \%$ de las vacas habían parido y tenían ternero al pie. Normalmente, la cantidad de 


\section{SUPLEMENTACIÓN CON MG EN VACAS CON RESTRICCIÓN ALIMENTARIA}

Mg circulante y disponible para el animal es de 2 a 2,5 g, pero durante la lactancia la excreción diaria de $\mathrm{Mg}$ por leche puede ser de 2,4 g lo cual duplica los requerimientos de este mineral (Surasak et al., 2004). Los valores de hipomagnesemia detectados en el G3 podrían explicarse teniendo en cuenta que la alimentación que recibían estos animales era heno (rollos) de pasturas de muy baja calidad obtenido de la propia R6.

La movilización de las reservas de Mg en el animal adulto ya sea desde el tejido óseo o muscular, es lenta y prácticamente nula. Esta situación determina una disminución en su concentración sanguínea y por ello es necesaria una ingesta continua en el momento del parto y al principio de la lactancia.

Las concentraciones de Ca y $\mathrm{P}$ disminuyen en el animal sano al momento del parto y durante la lactancia, luego dichos valores se recuperaron siendo esta situación fisiológica en el animal. Según Contreras (2002 b), si el descenso es intenso, debido a una incapacidad de movilización de $\mathrm{Ca}$ desde las reservas óseas, se podría producir paresia puerperal hipocalcémica.No se registraron signos clínicos de esta enfermedad si bien hubo hipocalcemia. Van de Braak et al. (1987) sostienen que concentraciones de $\mathrm{Mg}$ por debajo de $0,65 \mathrm{mmol} / \mathrm{L}$ en vacas en preparto incrementan la susceptibilidad a padecer hipocalcemia. Como ya se indicó, las concentraciones de Mg nunca estuvieron por debajo de ese valor lo cual podría explicar la falta de signos clínicos de paresia puerperal, a pesar de los bajos niveles de $\mathrm{Ca}$ observados en algunos casos. La misma dinámica de la calcemia en los tres grupos con valor mínimo en el posparto, coincide con el momento en el que los requerimientos nutricionales de $\mathrm{Ca}$ son elevados como consecuencia de la necesidad de una mayor cantidad de este mineral para la producción láctea. Si bien la hipocalcemia detectada fue importante, se podría especular que las vacas debieron reponer el Ca perdido removiendo al mismo desde los huesos o incrementando la absorción de este mineral a partir de la dieta (Jesse, 2006; Kronqvist et al., 2011).

Los animales no presentaron hipofosfatemia a pesar que la concentración de $\mathrm{P}$ en el alimento fue baja. Esto podría explicarse teniendo en cuenta que las vacas también tuvieron hipocalcemia subclínica. Se puede pensar entonces, que los mecanismos homeostáticos que regulan el metabolismo del $\mathrm{Ca}$ al remover este elemento de hueso también movilizaron $\mathrm{P}$, teniendo en cuenta que en el proceso de mineralización del hueso participan ambos minerales en una relación $\mathrm{Ca} / \mathrm{P}, 10 / 6$ (Thernout et al., 1985). El descenso de la fosfatemia encontrada en el G3 durante el muestreo 4 coincide con el momento del parto en animales que recibían un alimento de mala calidad sin ningún tipo de suplementación. Hadzimusic y Krnic (2012) también encontraron que los valores de $\mathrm{P}$ disminuyen no sólo con el estado fisiológico sino con la calidad del alimento que consumen los animales.

En cuanto al K, el hecho que su concentración en el alimento nunca fuera mayor al $2 \%$ probablemente hizo que no se presentaran casos de tetania.

Entre los factores que afectan la absorción de Mg desde el rumen, la concentración de $\mathrm{K}$ en la ración es el más importante (Martens, 2000; Phillips et al., 2006; Holtenius et al., 2008). Según Sykes (1993) concentraciones de $\mathrm{K}$ en la dieta superiores al 2\% MS, disminuyen la absorción de Mg.

Otra situación que fue favorable para que no hubieran manifestaciones clínicas de hipomagnesemia fue el valor del potencial tetanizante $\mathrm{K} / \mathrm{Ca}+\mathrm{Mg}$ puesto que el mismo es un factor determinante para la aparición de tetania (Brizuela et al., 2010).

Un elemento que ayuda a la absorción de $\mathrm{Mg}$ es el $\mathrm{Na}$. Los resultados encontrados para el $\mathrm{Na}$ en el muestreo 5 parecerían indicar que esta situación no afectó la absorción de Mg en el G1, posiblemente debido al hecho que el $\mathrm{Na}$ tiene mecanismos de regulación de la concentración en sangre muy eficaces. Sin embargo, en los G2 y G3 sí 


\section{CSEH, RODRÍGUEZ GARCÍA, SCIOTTIY CAMPERO}

se pudo observar que algunos animales presentaron hipomagnesemia. Un factor que pudo haber actuado favoreciendo la mejor absorción del Mg de los animales del G1 fue el suministro de maíz con el suplemento, el cual seguramente aportó energía a los animales de este grupo.

Las diferencias en el comportamiento del Mg extra e intracelular a lo largo de los muestreos marcan una disociación en su cinética intra y extra celular, donde posiblemente el animal trate de reestablecer la homeostasis intracelular, para luego regular el Mg circulante (Seeling y Berger, 1974). En este caso, el aumento del Mg intracelular que se observó en los tres tratamientos posparto estaría avalando esta aseveración.

Los resultados coinciden con los hallazgos de otros autores (Tongyai, 1987; Mulei y Daniel, 1988) quienes mencionan que, en bovinos, a diferencia de otras especies, la concentración de $\mathrm{Mg}$ en los glóbulos rojos es menor que en el suero. El hecho que los niveles de $\mathrm{Mg}$ en eritrocitos fuera constante cuando los niveles de Mg en suero tendieron a bajar se debe a que durante la carencia de $\mathrm{Mg}$, la disminución plasmática de este mineral es más rápida y marcada que la eritrocitaria (Cseh y Drake, 2000). Ello evidencia que en los bovinos, el $\mathrm{Mg}$ eritrocitario no es un buen indicador para la detección de deficiencias de $\mathrm{Mg}$ ya que aparentemente no existe una buena correlación entre el $\mathrm{Mg}$ en plasma y los niveles de $\mathrm{Mg}$ eritrocitario (Cseh y Ridao, 1994; Schweigel et al., 2009).

Para el caso del Ca, Na y K intracelular se podría especular que para detectar modificaciones importantes en las concentraciones intracelulares de estos cationes, posiblemente sea necesaria la presencia de severos cuadros de hipomagnesemia que alteren el equilibrio intra y extracelular de estos cationes. De hecho durante las crisis de tetania, el contenido de $\mathrm{Mg}$ en la circulación aumenta drásticamente debido al pasaje de $\mathrm{Mg}$ del compartimiento intracelular al extracelular (LBCV, 2010).
Las vacas gestantes evidencian períodos críticos en el último tercio de gestación y hasta el pico de lactancia (día 60 de lactancia), durante los cuales las necesidades de los minerales aumentan (Suttle, 2010). Si el alimento no provee de concentraciones adecuadas de los mismos, los animales pueden manifestar problemas sanitarios y disminución de los índices productivos como fue el caso de este trabajo. Por ello, es importante realizar una suplementación con Mg para evitar problemas de baja condición corporal, abortos, disminución del peso corporal, partos con problemas (Urdaz et al., 2003; Maresca et al., 2004; Elliot, 2009).

Los requerimientos maternos aumentan cuando se aproxima el parto llegando a su máximo a los 45-60 días posparto, debido a la producción de leche. El ternero durante los primeros meses de vida es casi exclusivamente lactante y depende de sus reservas corporales hasta los 2-3 meses de vida cuando comienza a desarrollar su rumen para completarlo a los 6 meses de vida. Los resultados de este trabajo, coinciden con los de Sager (1995), quien establece que si durante el final de la gestación y el periodo de lactación la madre es deficiente en minerales, los problemas se manifiestan en las crías con bajo porcentaje de destete, menor peso al destete y al nacimiento, mayor incidencia de natimortos y mayor susceptibilidad a las infecciones.

\section{CONCLUSIONES}

Si bien no se observaron diferencias significativas en los parámetros bioquímicos analizados, ni signos clínicos vinculantes con hipomagnesemia en los animales con restricción alimentaria temporaria, este grupo presentó mayores pérdidas productivas que los sin restricción.

Esta situación debería ser evaluada al momento de definir la estrategia de suplementación con $\mathrm{Mg}$ durante el preparto y la lactancia en animales sometidos a condiciones nutricionales similares a las de este trabajo. 


\section{SUPLEMENTACIÓN CON MG EN VACAS CON RESTRICCIÓN ALIMENTARIA}

\section{AGRADECIMIENTOS}

A la memoria de José Luis Pereyra. A los

\section{BIBLIOGRAFÍA}

Allworth, M.B., West, D.M. and Bruere, A.N. 1985. Magnesium status of dairy cows. New Zeal Vet J, 33: 171-172.

Bould, C. 1964. Magnesium in soils and plants. Vet Rec, 76: 1377-1381.

Brizuela, M.A., Ferri, C.M. y Cseh, S.B. 2010. Potencial tetanizante de verdeos de invierno en la Región Pampeana húmeda y semiárida. Rev Arg Prod Anim, 30: 239-240.

Capen, C.C. and Rosol, T. 1989. Calcium regulating hormones and diseases of abnormal mineral (calcium, phosphorus, magnesium) metabolism. In: Kaneko, J.J. (Ed.). Clinical Biochemistry of Domestic Animals. Academic Press. $4^{a}$ ed. San Diego. USA. pp. 678-752.

Carrillo, J. 1997. Manejo de un rodeo de cría. $2^{\underline{a}}$ ed. Ed. Centro Regional Buenos Aires Sur. 134 pp.

Contreras, P.A. 2002a. Hipomagnesemia: efectos y procedimientos de prevención en los rebaños. En: Alonso Díaz, A.J., González Montaña, J.R., Rejas López, J. Actas del Congreso de la Sociedad Española de Medicina Interna Veterinaria. Universidad de León. España. pp. 20-29.

Contreras, P.A. 2002b. Paresia puerperal hipocalcémica. Circular 139. <http://www. exopol.com/general/circulares/139.html.1-7> (05/12/2009).

Cseh, S.B. y Ridao, M. 1994. Dosaje de magnesio eritrocitario. Rev Arg Prod Anim, 14: 221- 225.

Cseh, S.B., Fay, P.J., Sueldo, R.J. y Drake, M.L. 1994. Una microtéctica simple para el dosaje de fósforo inorgánico en el suero bovino. Rev Arg Prod Anim, 14: 137-138.

Cseh, S.B.y Crenovich, H. 1996. Hipomagnesemia en el sudeste de la provincia de Buenos Aires, Argentina. Arch Med Vet, 28: 111-116.

Cseh, S.B. y Drake, M. 2000. Búsqueda de cambios en la composición mineral plasmática y eritrocitaria en bovinos deficientes en magnesio. 8ª Reunión Científico Técnica de la Asociación de Veterinarios de Laboratorio de Diagnóstico. San Luis. Argentina. pp. 80-84.

Chem, P.S., Toribara, T.Y. and Warner, H. 1959. Microdetermination of phosphorus. Annu Chem, ayudantes de campo de la Reserva Ganadera 6, del Inta Balcarce y a las auxiliares técnicas Maria Yarrar y Mónica Drake.

28: $1756-1758$.

David, A. and Morrow, D.V.M. 1986. Diagnosis, treament and prevention of reproductive diseases in small and large animals. Curr Therapy Theriogenology, 2: 320-341.

Elliot, M. 2009. Grass tetany in cattle-treatment and prevention. Department of Primary Industries. NSW. Primefact, 421. 4 pp.

Hadzimusic, N. and Krnic, J. 2012. Values of calcium, phosphorus and magnesium concentration in blood plasma of cows in dependence on the reproductive cycle and season. Istambul Univ Vet Fak Derg, 38: 1-8.

Holtenius, K., Kromqvist, C., Briland, E. and Spörndly, R. 2008. Magnesium absorption by lactating dairy cows on a grass silage based diet supplied with different potassium and magnesium levels. J Dairy Sci, 94: 743-748.

Jesse, P. 2006. Macromineral physiology and application to the feeding of the dairy cow for prevention of milk fever and other periparturient mineral disorders. Anim Feed Sci Tech, 126: 237-257.

Kemp, A. and t'Hart, M.L. 1957. Grass tetany in grazing milk cows. Neth J Agr Sci, 5: 4-7.

Kronqvist, C., Emanuelsen, U., Spörndly, R. and Holtenius, K. 2011. Effects of prepartum dietary calcium level on calcium and magnesium metabolism in peripartum dairy cows. $J$ Dairy Sci, 94: 1365-1373.

Langlands, J.P. 1974. Studies on the nutritive value of the diet selected by grazing sheep. A note on hand plucking as a technique for estimating dietary composition. Anim Prod, 19: 249-251.

Laporte, J.A. and Sykes, A.R. 2004. Intestinal magnesium absorption in ruminant compensate by reduction ruminant absorption. XI Congress International Society of Animal Clinical Biochemestry. Valdivia. Chile. pp. 47.

LBCV. 2010. Laboratroio de Bioquímica Clínica Veterinaria del Instituto Nacional de Tecnología Agropecuaria. Base de datos. INTA Balcarce. Argentina. 


\section{CSEH, RODRÍGUEZ GARCÍA, SCIOTTIY CAMPERO}

Ledesma, A. 2008. Evaluación de la magnesemia en un rodeo de cría incluido en un sistema integrado de manejo pastoril. Tesis de grado. Fac. Cs. Vet. UNPBA. Tandil. 45 pp.

Maresca, S., Quiroz García, J.L. y Cseh, S.B. 2004. Efecto de la suplementación con óxido de magnesio sobre la concentración plasmática de magnesio en vacas de cría. Rev Arg Prod Anim, 2: 375-376.

Martens, H. and Schweigel, M. 2000. Pathophysiology of grass tetany and other hypomagnesemias implications for clinical managent. Vet Clin NAnFood A, 16: 339-368.

Merck, 2003. Merck veterinary manual. $8^{\text {th }}$ ed. Merck Co. Inc. Wdjitehause Station. NJ. USA. 1907 pp.

Mulei, C.M. and Daniel, C.W. 1988. The effect of induced hypomagnesemia and hypermagnesaemia on the erythrocyte magnesium concentration in cattle. Vet Res Commun, 12: 289-293.

NRC. 2000. National Research Council. Nutrient requirements of beef cattle. $7^{\text {th }}$ edition. National Academic Press. Washington, D.C. USA.

Odette, O. 2005. Grass tetany in a herd of beef cows. Can Vet J, 46: 732-734.

Perkin Elmer. 1982. Analytical methods for atomic absorption spectrofotometry. The Perkin Elmer Corporation Conneticut. USA. pp. 410.

Phillips, C.J.C., Mahomed, M.O. and Chiy, P.C. 2006. The critical dietary potasium concentration for induction of mineral disorders in nonlactacting Welsh Mountain sheep. Small Ruminant Res, 63: 32-38.

Sager, R.L. 1995. Suplementación mineral en bovinos para carne en la cría del siglo XXI. Difusión Ganadera, 8: 175-200.

SAS/STAT. 1995. User's (Release 6.03) SAS. Inst. Inc., Cary, NC. pp.1-277.

Scandolo, D. 2005. Evaluación física, química y biológica de productos de magnesio para la suplementación de vacas lecheras a pastoreo. Tesis de Magister. Facultad de Ciencias Vete- rinarias. Universidad Austral de Chile.

Seeling, M.S. and Berger, A.R. 1974. Range of normal serum magnesium values. New Engl $J$ Med, 290: 974-975.

Schweigel, M., Voigt, J. and Mohr, E. 2009. Indication of intracellular magnesium deficiency in lactating dairy cows revealed by magnesium loading and renal fraction excretion. J Anim Physiol An N, 93: 105-112.

Surasak Jittakhot, J., Schonewille, T., Wouterse, H., Focker, E.J., Yuangklang, C. and Beynen, A.C. 2004. Effect of high magnesium intake on apparent magnesium absorption in lactating cows. Anim Feed Sci Tech, 113: 53-60.

Suttle, N.F. 2010. Mineral nutrition of livestock. $4^{\text {th }}$ Edition. CABI. Amazon.co.uk. UK. 587 pp.

Sykes, A.R. 1993. Hipomagnesemia en bovinos. $1^{\text {a }}$ Jornadas Chilenas de Buiatría. Actas de Osorno. Chile. pp. 117-127.

Thernout, J.H., Davies, H.M.S., Milton, J.T.B., Simpsom-Morgan, M.W. and Sands, N. 1985. Phosphorus metabolism in rumiants. ITechniques for phophorus depletion. Aust J Agr Res, 36: 637-645.

Tongyai, S. 1987. Mise en évidence d'une modification de la fluidité membranaire des erythrocytes au cours de la carence expérimentale en magnesium, chez le rat. Thèse Docteur de $3^{\text {èrme }}$ cycle. Université de Clermont II. France. pp. 171-180.

Urdaz, J.H., Santos, J.E., Jardon, P. and Overton, M.W. 2003. Importance of appropriate amounts of magnesium in rations for dairy cows. $J A m$ Vet Med Assoc, 222: 1518-1523.

Van de Braak, A.E., Van'T Klooster, A.T. and Malestein, A. 1987. Influence of a deficient supply of magnesium during the dry period on the rate of calcium mobilisation by dairy cows at parturition. Res Vet Sci, 42: 101-108.

Whitaker, D.A. 2000. Use and interpretation of metabolic profils in dairy cows. In: Andrews, A.H. (Ed). The health of dairy cattle. Blackwell Sciencie. Oxford. UK. pp. 89-107. 